\title{
How future-proof is the Continental European frequency control
}

\section{structure?}

\section{Conference Paper}

\section{Author(s):}

Scherer, Marc; Andersson, Göran

Publication date:

2015

Permanent link:

https://doi.org/10.3929/ethz-b-000105094

Rights / license:

In Copyright - Non-Commercial Use Permitted

Originally published in:

https://doi.org/10.1109/PTC.2015.7232604 


\section{How Future-Proof Is the Continental European Frequency Control Structure?}

\author{
Marc Scherer \\ Swissgrid Ltd., Switzerland \\ marc.scherer@swissgrid.ch
}

\author{
Göran Andersson \\ ETH Zürich, Switzerland \\ andersson@eeh.ee.ethz.ch
}

\begin{abstract}
Recent studies have discussed the operational challenges in the European power system which come along with the increasing penetration of intermittent generation in the coming years. Based on time sequential Monte Carlo simulations, this paper investigates to what extent a decentralized frequency control structure is appropriate with respect to three European scenarios. To evaluate the future adequacy of the current control structure and its active power reserves, a reduced simulation model, which determines the frequency quality in Continental Europe, is presented. The results reveal that the control framework is technically robust, but either the active power reserves need to be significantly increased by end-2020 to sustain the current realtime approach or the frequency control policy must be changed in favor of retrospectively averaging frequency deviations by means
\end{abstract} of joint control actions.

Index Terms-20-20-20 targets, active power reserves, adequacy, frequency control, time control.

\section{INTRODUCTION}

The last decade brought major changes and challenges to the energy policy debate. How do these organizational and structural changes affect the present processes of real-time matching of electricity generation and consumption in the long run? Certainly, the physical basis for frequency control will not change, and the decentralized control structure is well-established in the Continental European power system, but the slowly continuing reshaping of the power system challenges the adequacy of the available resources of frequency control.

There are several drivers that affect the long-term frequency quality, i.e. the frequency control adequacy:

- Intermittent generation, such as wind and solar power, has been adding volatility and operational complexity to the system over the past years [1], [2].

- The generation structure is changing from centralized large conventional units to distributed production; this is underlined by the decision of several countries to abandon nuclear power [3].

- The increase in electricity trading implies a schedulebased grid operation instead of classic real-time load following, and further, most system operators only have access to a predefined amount of reserves [4].

- The on-going liberalization has put system operators under increased cost and efficiency pressure [5], [6].

Nowadays, national system operators are responsible for frequency control either as Independent System Operators
(ISOs), who operate only, or as Transmission System Operators (TSOs), who operate and own the transmission system. Active power reserves for frequency restoration are assessed and dimensioned according to national rules, standards and regulatory authorities [7]. This raises the question of the overall frequency performance. In this context, the contribution of this paper is twofold: Quantifying the long-term adequacy of current frequency control resources and outlining the impact of the generation mix and load evolution according to different European scenarios. We rely on a reduced power system model for a steady state frequency analysis, and utilize time sequential Monte Carlo simulations, which are a common approach to reliability modeling [8].

This paper is organized as follows: Section II outlines an approach for reducing the frequency control framework and standardizing frequency control resources. Section III presents the simulation model and the scenarios. Section IV provides the results. Finally, Section V is devoted to conclusions and perspectives.

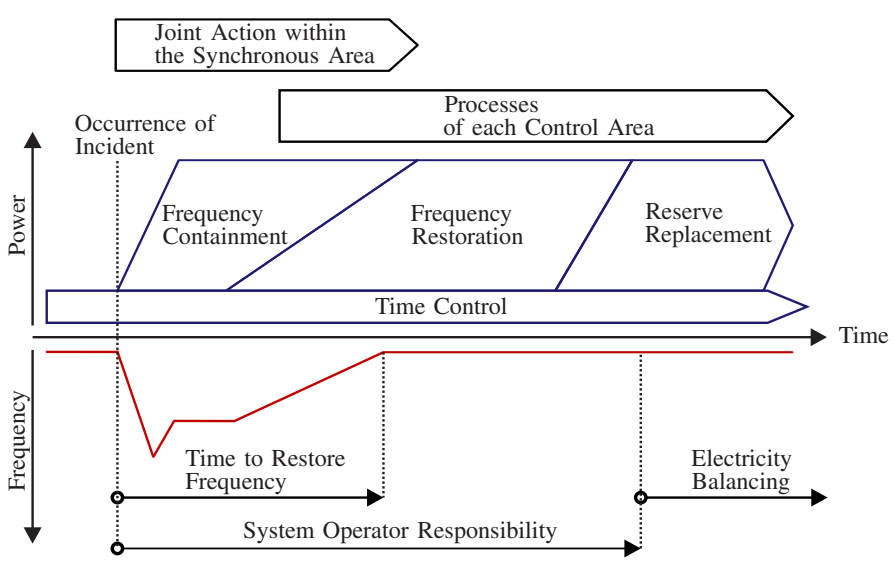

Fig. 1: Hierarchy and chronology of frequency control.

\section{Reduced Frequency CONTROL Framework}

\section{A. Frequency Control Processes}

In most large interconnected power systems, frequency control exhibits a four-level hierarchy which involves frequency containment ("primary control"), frequency restoration ("secondary control"), reserve replacement ("tertiary control"), and time control. Figure 1 illustrates these control processes. 
All but time control are triggered by single incidents. Time control is a common process by which system operators jointly compensate for the accumulated daily frequency deviation.

As we investigate the capability in the Continental European power system to cover imbalances between supply and load, we are interested in neither the short-term dynamics after the occurrence of an imbalance nor the national differences in electricity balancing markets, i.e. balance responsible party behavior; instead, we focus on the steady states in which the power system can exist considering standard conditions. Such a steady state consideration allows for a lower time resolution in favor of a large simulation time horizon.

\section{B. Steady State Consideration}

In order to analyze the long-term impact of changes in load and the generation portfolio on frequency control, we need to consider the basic frequency control structure and the amount of available active power reserves in a liberalized market environment, where these reserves are split into a number of ancillary service market products which differ from country to country. As we focus on the overall frequency control adequacy, we assume that every control area, i.e. country, is meant to perform equally within the time to restore frequency, which is 15 minutes in Continental Europe [7]. In addition to this, the national settlement period ("imbalance period") in most European countries is not equal to the time to restore frequency: Depending on the market design, schedule notifications have a granularity of 15 minutes, 30 minutes, or one hour. In light of increasing electricity trading, this can cause market-induced load-generation imbalances [4]. The severity of this issue and, therefore, the contribution to market-induced imbalances depends on the national design of electricity balancing markets, its imbalance pricing, and the available operational mitigation measures. Thus, we will not take into account the impact of market-induced imbalances, but rather, give preference to a time sequential model with an hourly time resolution. With this, we decouple the question of product design ("standard products"), degree of liberalization, and national differences in system operation from long-term frequency control quality, i.e. the ability of the entire system to compensate for an imbalance.

\section{Model For Continental Europe}

Our objective is to investigate the frequency control adequacy by simulating the long-term frequency quality in the Continental European power system. Figure 2 outlines the basic simulation framework.

\section{A. Scenarios and Historical Data}

As we target a realistic design, we use the ENTSO-E system adequacy outlook data to parameterize the simulation framework [9]. It features three scenarios which provide reference points for future load, and wind as well as solar power production. Scenario A and B are both bottom-up scenarios which are based on the expectations of the system operators. Scenario A assumes a rather conservative investment behavior; it takes into

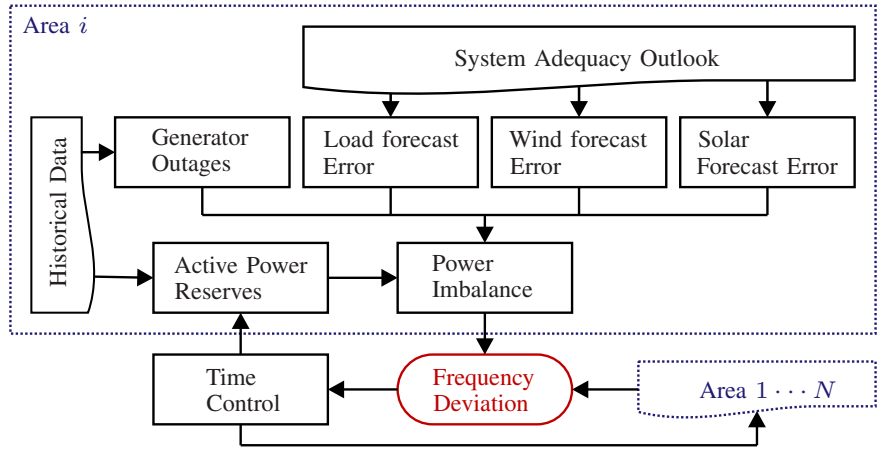

Fig. 2: Simulation framework for the Continental European power system.

account only the generating capacity developments which are considered secured. Scenario B represents the best estimate provided that market signals give adequate incentives for investments. Scenario C ("Scenario 20-20-20") is derived from the National Renewable Action Plans (NREAP) in compliance with the 20-20-20 targets of the European Union for 2020 in climate and energy policy, i.e. $20 \%$ reduction in greenhouse gas emissions at 1990 levels, $20 \%$ increase in renewable energy as a share of the total energy production, and $20 \%$ reduction in energy consumption at projected 2020 levels by improving energy efficiency.

\section{B. Generation Portfolio}

The most obvious reason for large imbalances are forced equipment outages. Due to the high degree of meshing of the Continental European power system, this can be reduced to forced generation unit outages. Failure statistics to calculate those forced outages are based on historical data [4]. The outages are modeled by a two-state Markov process [8].

In order to avoid a systematic overassessment of imbalances due to the tripping of single units, we consider the degree of utilization: If demand falls below the amount of intermittent and nuclear generation, the latter is accordingly curtailed. Otherwise, the remaining share is allocated according to a merit order from least to most expensive respecting minimum load factors, i.e. hydro (subject to maximum full load hours), lignite, coal, gas, and oil.

\section{Forecast Errors}

The net forecast error results from load as well as wind and solar power production.

1) Load: Load forecast errors can be well-modeled by a normal distribution. Seasonal hour-ahead load forecast statistics for the California Power Systems indicate an average standard deviation of $1.41 \%$ of the load and a negligible mean value [2]. The resulting values are supported by corresponding European statistics, which assume even slightly higher values [10]. They appear to remain quite stable over time, in particular when compared to pre-liberalization data [11].

Regarding future development, it can be argued that enhanced demand side management that decreases load fore- 
casting uncertainty runs counter to the increasing uncertainty due to the additional use of standard load profiles for domestic customers in liberalized markets; thus, the load forecast error is assumed to remain stable in the next years.

2) Wind: A wind power forecast error depends not only on the weather forecast but also on the geographical dispersion and the number of wind turbines. Its forecast errors, in particular for non-specific locations, are often assumed to be normally distributed [1]. Lately, more sophisticated distributions have been proposed to more accurately model the semi-heavy tails observed with typical statistical data [12]. Therefore, we rely on a (generalized) hyperbolic distribution which allows not only for a mean value and a standard deviation but also for a skewness as well as a kurtosis. To obtain a general model, we analyzed a given data set of wind power forecasting errors from six countries all across Europe, including Germany and Spain [13]. We found a good linear fitting, which can be described by a regression coefficient and an intercept, between installed wind capacity and all three mean value, standard deviation, and kurtosis, as illustrated in Figure 3. No significant relationship could be found for the skewness, which is assumed to be zero.

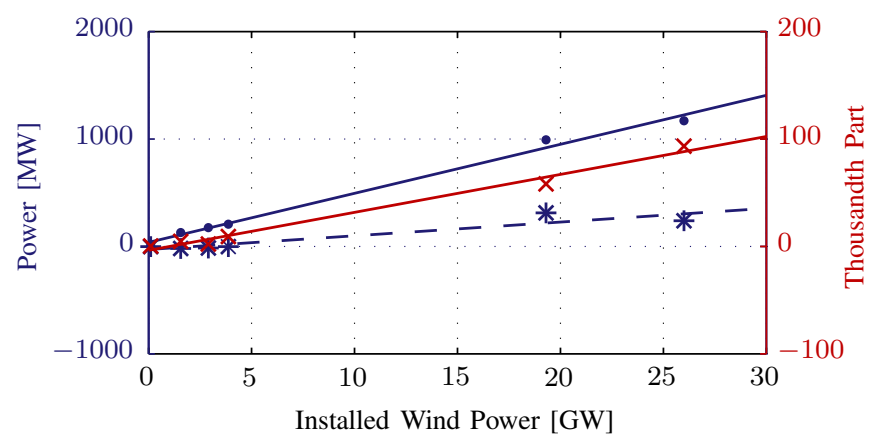

$\longrightarrow$ Standard Deviation $\quad$ * $\longrightarrow$ Mean Value $\longrightarrow$ Kurtosis

Fig. 3: Linear extrapolation to determine mean value, standard deviation, and kurtosis as a function of the installed wind power capacity.

Such a generalization is subject to uncertainty: Notwithstanding the political ambition of the European Union concerning the promotion of renewable energy, the national strategic and political endeavors vary: To what extent does this approach encompass changes in the forecasting quality? Spain and Germany are the countries with the largest share of wind power generation in Europe, and they are considered to have assumed a vanguard role in dealing with the challenges associated with a high penetration of intermittent generation, which includes advanced forecasting experience and techniques. Therefore, the linearization presented in Figure 3 is assumed to consider the increasing relative forecasting quality in a rudimentary way: The relative quality improves, the more wind power generation is installed.

3) Solar: Characteristics of the solar power forecast errors have not yet been sufficiently well studied, and they are neither completely random nor completely deterministic [1]. For the short-term variations, conformities to wind power forecasts exist during daytime. Under the assumption of the absence of heat storages for solar thermal power, solar power is negligible during the night; hence, its forecast error is assumed to be zero. Unlike for wind power, the degree of utilization for solar power exhibits a distinctive daily and annual pattern.

The solar power forecast error during daytime is assumed to be normally distributed as a function of the degree of utilization. The mean value is zero. The scaling of the standard deviation is similar to that of wind power. For each country $i$, the standard deviation $\sigma$ of the solar forecast error is a function of the solar power production $P_{P V}^{i}$ and the total installed capacity $P_{P V, t o t}^{i}$ :

$$
f_{\sigma}^{i}\left(P_{P V}^{i}, P_{P V, t o t}^{i}\right)=\frac{a_{\sigma} \cdot P_{P V, t o t}^{i}+b_{\sigma}}{P_{P V, t o t}^{i}} \cdot P_{P V}^{i}
$$

In order to determine $P_{P V}^{i}$, we rely on a typical seasonal profile, which has been determined from historical load factors between 2009 and 2011.

\section{The Time Control Process}

Time control is meant to correct deviations between electrical time and Coordinated Universal Time (UTC, also used as a backronym for Universal Time Coordinated). This service is essential for equipment that relies on the frequency for timekeeping. These are often legacy devices such as electrical meters, electric clocks, and electro-mechanical street light timers as well as controls.

As the instantaneous frequency is corrected to its nominal value after an incident without a compensation of the accumulated energy mismatch, time control also sustains the long-term energy balance in the system: The energy for frequency containment, i.e. primary control energy, is not explicitly settled in Continental Europe, and the providers profit considerably from a zero average frequency and the respective planning certainty.

The electrical time depends on the frequency (or voltage) time period, which is $20 \mathrm{~ms}$ for $50 \mathrm{~Hz}$; thus, the instantaneous frequency $f_{i}$ over a given time $t$ determines the time error $\epsilon$ :

$$
\epsilon=\int_{0}^{t} \frac{f_{i}-50 \mathrm{~Hz}}{50 \mathrm{~Hz}} \mathrm{~d} t
$$

The time error is used for real-time monitoring and serves as a performance indicator. If the daily time error exceeds $\pm 20 \mathrm{~s}$ in the Continental European power system, the frequency setpoint is adjusted by $\mp 10 \mathrm{mHz}$ for the whole next day. The activation dead band of frequency containment is also $10 \mathrm{mHz}$ for time control to generally not trigger frequency-response reserves, since for those the frequency set-point usually cannot be adjusted.

Time control is monitored and coordinated by one dedicated system operator ("time monitor") in the synchronous area. The target range in normal operation for the maximum time error is $\pm 30 \mathrm{~s}$. 


\section{E. Initial Active Power Reserve Obligation}

The availability as well as the activation dynamics of the active power reserves determine the means of a system operator to control imbalances and thus its contribution to the frequency quality. The dynamic behavior of both the control loop and the providing units can be neglected for the long-term frequency assessment. Due to steady state considerations, the difference between automatic and manual reserves may only be considered rudimentarily.

The results for 2010 of a probabilistic reserve dimensioning approach serve as a basis for scaling the amount of the available reserves for each Continental European country [6]. This study assumes a deficit probability of $0.1 \%$; however, in reality active power reserves are dimensioned less conservatively [7]. Therefore, the given amounts of reserves are uniformly scaled for all countries in order to minimize the Mean Squared Error (MSE) of the frequency's standard deviation, that is, the difference between historical and modeled frequency between 2010 and 2013. Due to time control, the mean value is obviously zero for the current situation.

\section{ANALYSIS AND EVALUATION}

\section{A. Model Validation}

The model is first tested for its robustness. The validation focuses on the years 2010 to 2013. The robustness of the model could be shown by a detailed frequency analysis. Table I summarizes the frequency range analysis.

\begin{tabular}{lllll}
\hline & 2010 & 2011 & 2012 & 2013 \\
\hline Measured & $10.95 \mathrm{mHz}$ & $9.54 \mathrm{mHz}$ & $10.56 \mathrm{mHz}$ & $10.88 \mathrm{mHz}$ \\
Modeled & $9.39 \mathrm{mHz}$ & $9.91 \mathrm{mHz}$ & $10.79 \mathrm{mHz}$ & $11.16 \mathrm{mHz}$ \\
\hline
\end{tabular}

TABLE I: Comparison between historical and simulated standard deviations.

Overall, there is a good matching of the heavy tail behavior, whereas the measured frequency shows a slightly higher kurtosis than the one determined by the model, which affects small values roughly around $\pm 2.5 \mathrm{mHz}$ as illustrated in Figure 4.

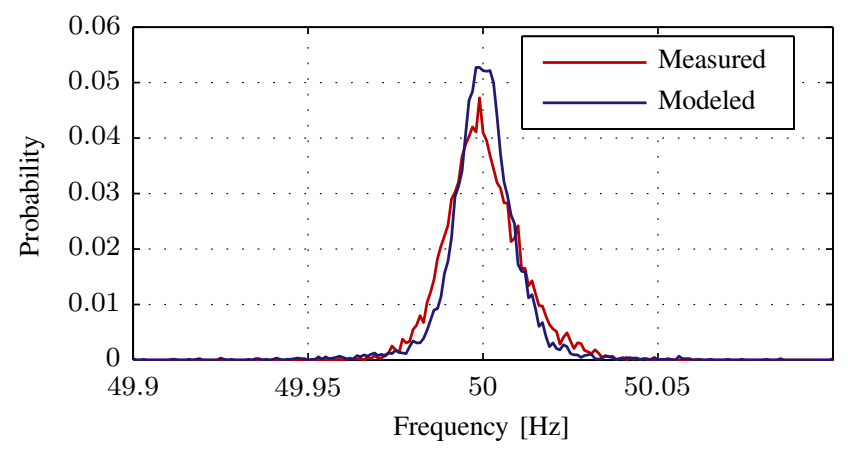

Fig. 4: Frequency validation for 2013.

\section{B. Time Error}

The electrical time deviation is a robust indicator of the overall trend of the frequency quality: One might speculate that the accumulated time error should oscillate within certain boundaries with a mean value close to zero. However, the results illustrated in Figure 5 show a different trend. A clear negative drift of the accumulated time error is observable for all three scenarios. The most significant time difference is expected for Scenario C with a time error of $1.5 h$ by end-2020. The negative time drift implies an average frequency below the nominal value, which is mainly caused by an overestimation of wind and solar power production. Until 2016, all three scenarios exhibit a similar time difference. However, after that, the scenarios start to drift apart. It can be concluded that the time difference is increasing with the expansion of wind and solar power capacity if the reserve resources are not adjusted accordingly.

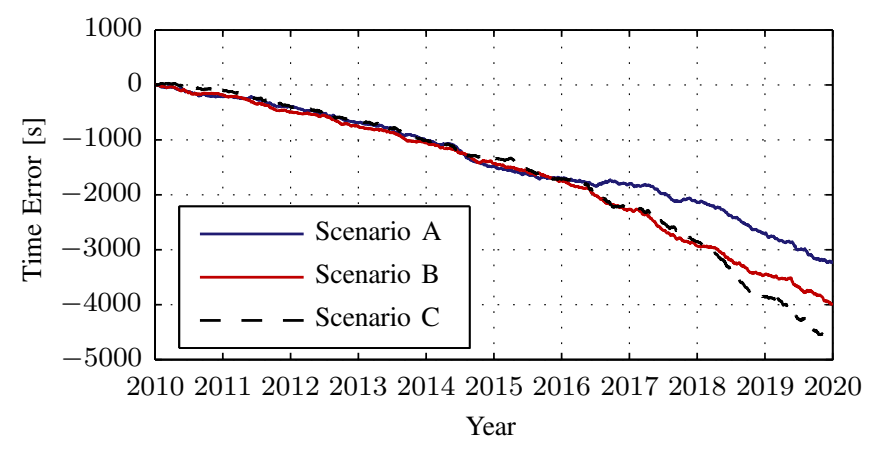

Fig. 5: Time error analysis (without the activation of time control).

As a consequence, we need to inquire into the extent to which time control is able to avoid such a long-term energy mismatch in the system. Figure 7 summarizes the resulting performance of time control by end-2020. The three scenarios exhibit a comparable and low average time error, which indicates a stable average frequency. However, the maximum target discrepancy cannot always be ensured. Scenarios A, B and $\mathrm{C}$ exceed the limit of $\pm 30 \mathrm{~s}$ for 13,15 , and 39 days, respectively. Obviously, Scenario $\mathrm{C}$ challenges time control the most; in particular, negative time correction increases significantly.

\section{Frequency Quality Evolvement}

Time control is an adequate means to sustain the desired long-term of $50 \mathrm{~Hz}$, but how does the instantaneous frequency evolve, and how is it affected by the time correction? In normal operation, the frequency is supposed to stay within the hourly standard frequency range of $10 \mathrm{mHz}$ [7]. To analyze the frequency quality, and the potential interference with time control in particular, we calculate the numbers of hours in which the frequency violates the standard frequency range for both the current control setup and one without time control.

In the conservative Scenario A, the proportion of time above and below the standard frequency range increases by $65.5 \%$ 


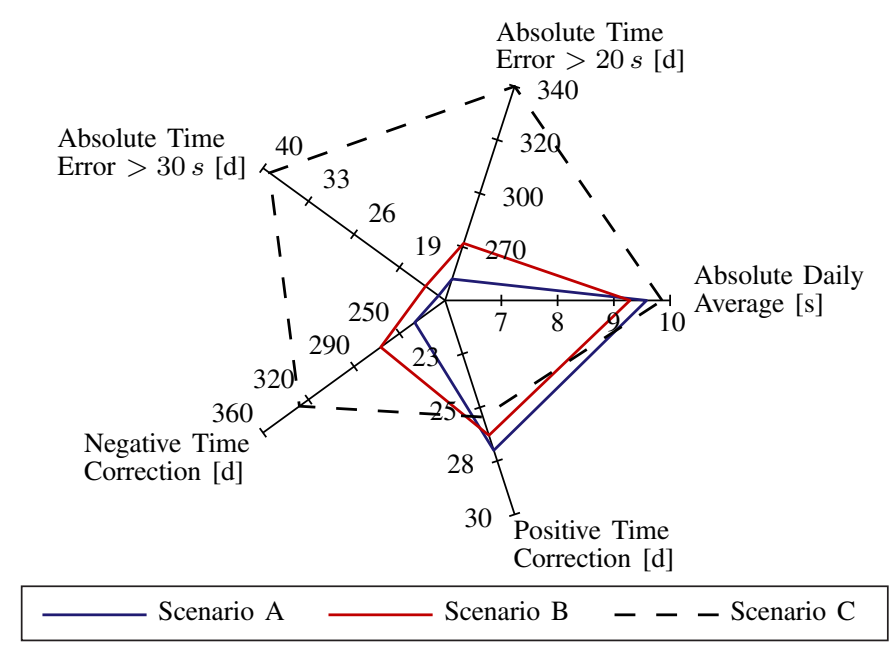

Fig. 6: Time control performance by end-2020.

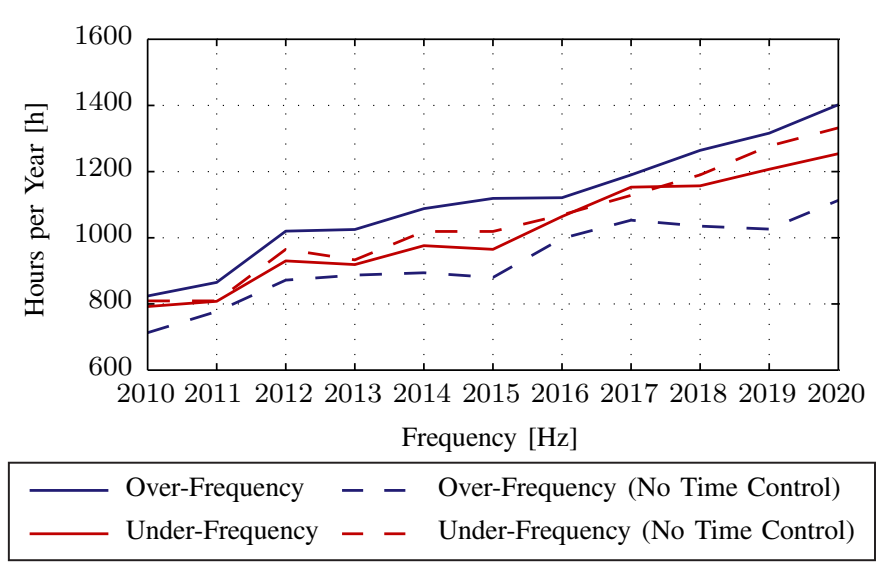

Fig. 7: Over-frequency and under-frequency evolvement with as well as without time control.

and $58.3 \%$, respectively; thus, the frequency quality will decrease. It is strongly influenced by the changes in production, and the share of intermittent generation in particular. The time correction itself does, however, not necessarily contribute to this trend: In the absence of time control, the frequency is even more likely to violate the standard frequency range by under-frequency.

\section{Active Power Reserve Sensitivity}

Until now, we assumed no changes in the amount of active power reserves, as the dimensioning is typically static [7]. As shown above, the frequency quality will significantly change in the upcoming years if the amount of active power reserves is not adjusted. The necessary increase in active power reserves to ensure a stable standard frequency deviation of $10 \mathrm{mHz}$, i.e. the hourly standard frequency range, is shown in Figure 8. In Scenarios A, B and C, the active power reserves need to be increased yearly by $2.5 \%, 3.2 \%$ and $4.7 \%$ on average, respectively. Furthermore, to achieve the 20-20-20 targets, the active power reserves need to be increased by $41 \%$ between 2014 and 2020.

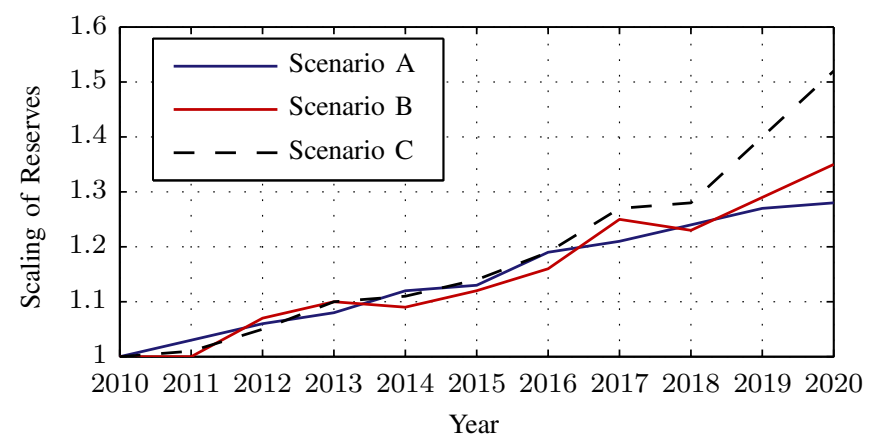

Fig. 8: Increasing reserve requirements for a constant hourly frequency standard deviation of $10 \mathrm{mHz}$ (Scenario A in 2010 serves as a reference point).

The growing share of intermittent generation obviously causes the increasing need for active power reserves. Figure 9 shows the weekly maximum and minimum of the load and wind power and solar power forecast errors for 2013 as well as 2020 , i.e. the total imbalances for all European countries have been summed up. For the sake of clarity, forced unit outages are not plotted; their likelihood of occurrence stays the same. In 2013, load is the predominant factor for the overall imbalance, and only a very minor seasonal pattern can be observed for wind and solar power forecast errors. In 2020, wind and solar power mainly determine the overall imbalance. A seasonal pattern can be spotted for wind and solar power forecast errors. The latter has a remarkable impact in the summer season; it even partly transcends the load forecast error. Thus, load is changing from being the predominant influencing factor for the need for active power reserves to a minor but stable one. This confirms earlier studies that investigated the limitations of load-related and deterministic reserve dimensioning approaches [14].

\section{Closing Remarks}

\section{A. Conclusion}

Although the European legislation, driven in particular by the vision of a pan-European market, is pushing towards a consolidation of the European electricity sector, the supervision structure for system operation has been kept in its original form, where primarily national system operators supervise their respective part of the system, and thus manage frequency control decentrally. In this context, the contribution of this paper is twofold: First, we showed the future robustness of the present Continental European frequency control approach. Second, we confirmed the increasing demand for active power reserves for this approach in the course of the next years: Load as the main driver for active power reserve dimensioning will be superseded by the overall generation structure, i.e. intermittent generation will be the crucial future factor.

We outlined a reduced frequency control simulation model, and used the ENTSO-E system adequacy outlook data to simulate the Continental European frequency behavior until end-2020. With that, we demonstrated the effect on frequency 


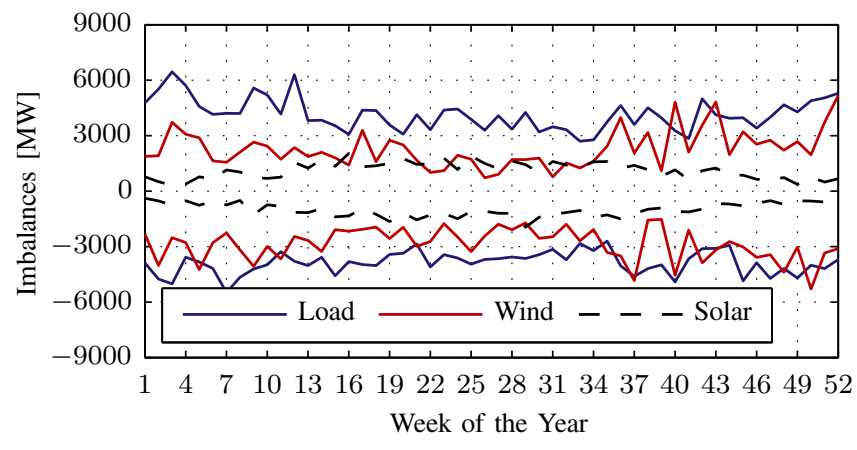

(a) Maximum and minimum weekly imbalances in 2013.

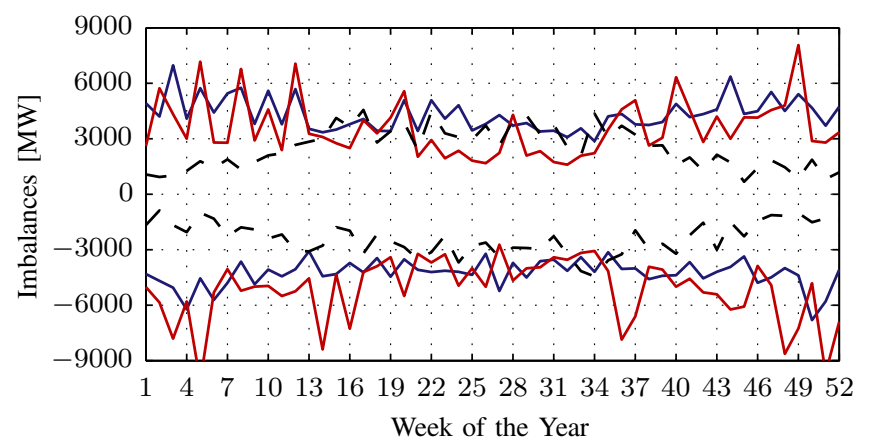

(b) Maximum and minimum weekly imbalances in 2020 (same legend as in Figure 9a).

Fig. 9: Load, wind power and solar power forecast errors.

control quality and resources in light of three possible European development scenarios. The overall impact is quite independent of the scenario: Only a progressive scenario will strongly increase the needed resources after 2018 and, therefore, challenge a decentralized frequency control structure.

Time control as a common control process, whose raison d'être has been called into question in the past years, will gain importance, especially if active power reserves are not accordingly increased. If so, there will be a shift from nationally compensating for imbalances in real-time to retrospectively averaging frequency deviations by means of time control, which would imply a change in the European frequency control policy. In simple terms, the granularity of the adherence to the nominal frequency will diminish.

In terms of costs, the current control structure can be seen from two angles: Without an increase in costs, it still provides the means for a sustainable long-term frequency quality, but the current costs-by-cause principle, i.e. principles of noninteractive control, will no longer apply as the compensation is shared across Europe. To preserve the established operational mode, additional costs up to roughly $50 \%$ of the current ones need to be accepted by all system operators.

\section{B. Outlook}

In the future, the system operators have to decide on the trade-off between operational sovereignty and cost efficiency: Most responsibilities, such as congestion management, operational planning, and ancillary services, are assigned to national system operators and their respective regulatory authority. A consolidation of existing control areas in order to access synergy effects is an obvious technical solution to counter an increasing demand for active power reserves. But this implies an internationalization of operational responsibilities; thus, a stepping up of efforts to share a larger amount of active power reserves among counties is more likely. The European work in progress "Guidelines" and "Network Codes" will support this proposition, which is underlined by concepts such as imbalance netting and common merit order lists. In this context, intermittent generation should be incentivized to gain operational flexibility or, at least, limit precedence in order to bound the respective forecast errors. This, however, raises doubts concerning the applicability of the current quality indicators and threshold values, which had heretofore been imposed symmetrically.

\section{ACKNOWLEDGMENT}

For his valuable contribution, the authors would like to thank Manuel Mühlebach (Swissgrid Ltd.).

\section{REFERENCES}

[1] Y. V. Makarov, S. Lu, N. Samaan, Z. Huang, K. Subbarao, P. V. Etingov, J. Ma, R. P. Hafen, R. Diao, and N. Lu, "Integration of Uncertainty Information into Power System Operations," in IEEE Power and Energy Society General Meeting, July 2011, pp. 1-13.

[2] Y. V. Makarov, C. Loutan, J. Ma, and P. de Mello, "Operational Impacts of Wind Generation on California Power Systems," IEEE Transactions on Power Systems, vol. 24, no. 2, pp. 1039-1050, May 2009.

[3] K. Bruninx, D. Madzharov, E. Delarue, and W. D'haeseleer, "Impact of the German nuclear phase-out on Europe's electricity generation," in 9th International Conference on the European Energy Market (EEM), May 2012, pp. 1-10.

[4] M. de la Torre Rodríguez, M. Scherer, D. Whitley, and F. Reyer, "Frequency containment reserves dimensioning and target performance in the European power system," in IEEE PES General Meeting Conference Exposition, July 2014, pp. 1-5.

[5] J.-M. Glachant, M. Saguan, V. Rious, and S. Douguet, "Incentives for investments: Comparing EU electricity TSO regulatory regimes," European University Institute, Tech. Rep., December 2013.

[6] M. Scherer, M. Zima, and G. Andersson, "An integrated pan-European ancillary services market for frequency control," Energy Policy, vol. 62, pp. 292-300, 2013.

[7] OpHB-Team, "Policy 1: Load-Frequency Control and Performance," in UCTE Operation Handbook, March 2009.

[8] R. Billinton and W. Li, "Reliability assessment of electric power systems using monte carlo methods," Plenum Press, 1994.

[9] ENTSO-E, "Scenario Outlook \& Adequacy Forecast (SO\&AF) 20132030," www.entsoe.eu, April 2013.

[10] C. Maurer, S. Krahl, and H. Weber, "Dimensioning of secondary and tertiary control reserve by probabilistic methods," European Transactions on Electrical Power, vol. 19, pp. 544-552, 2009.

[11] L. Söder, "Reserve margin planning in a wind-hydro-thermal power system," IEEE Transactions on Power Systems, vol. 8, no. 2, pp. 564 571, May 1993.

[12] H. Bludszuweit, J. Dominguez-Navarro, and A. Llombart, "Statistical analysis of wind power forecast error," IEEE Transactions on Power Systems, vol. 23, no. 3, pp. 983-991, Aug 2008.

[13] B.-M. Hodge, D. Lew, M. Milligan, H. Holttinen, S. Sillanp, E. GmezLzaro, R. Scharff, L. Sder, X. Larsn, G. Giebel, D. Flynn, and J. Dobschinski, "Wind Power Forecasting Error Distributions: An International Comparison," in The 11th Annual International Workshop on LargeScale Integration of Wind Power into Power Systems as well as on Transmission Networks for Offshore Wind Power Plants, November 2012.

[14] K. R. Voorspools and W. D. Dhaeseleer, "Are deterministic methods suitable for short term reserve planning?" Energy Conversion and Management, vol. 46, no. 13-14, pp. 2042-2052, 2005. 